\title{
Enhancing Cognition in Older Persons with Depression or Anxiety with a Combination of Mindfulness-Based Stress Reduction (MBSR) and Transcranial Direct Current Stimulation (tDCS): Results of a Pilot Randomized Clinical Trial
}

\author{
Heather Brooks ${ }^{1}$. Hanadi Ajam Oughli² $\cdot$ Lojine Kamel $^{2}$. Subha Subramanian² Gwen Morgan $^{3}$. \\ Daniel M. Blumberger ${ }^{1}$. Jeanne Kloeckner ${ }^{2}$ - Sanjeev Kumar ${ }^{1}$ - Benoit H. Mulsant ${ }^{1}$ • Eric J. Lenze ${ }^{2}$. Tarek K. Rajji ${ }^{1,4}$
}

Accepted: 18 September 2021 / Published online: 5 October 2021

(c) The Author(s), under exclusive licence to Springer Science+Business Media, LLC, part of Springer Nature 2021

\begin{abstract}
Objectives Individuals with subjective memory complaints and symptoms of depression and/or anxiety are at high risk for further cognitive decline, and possible progression to dementia. Low-burden interventions to help slow or prevent cognitive decline in this high-risk group are needed. The objective of this study is to assess the feasibility of combining MindfulnessBased Stress Reduction (MBSR) with transcranial direct current stimulation (tDCS) to increase putative benefits of MBSR for cognitive function and everyday mindfulness in depressed or anxious older adults with subjective cognitive decline. Methods We conducted a two-site pilot double-blind randomized sham-controlled trial, combining active MBSR with either active or sham tDCS. The intervention included weekly in-class group sessions at the local university hospital and daily at-home practice. Anodal tDCS was applied for 30 min during MBSR meditative practice, both in-class and at-home. Results Twenty-six individuals with subjective cognitive complaints and symptoms of depression and/or anxiety were randomized to active $(n=12)$ or sham tDCS $(n=14)$. The combination of MBSR and tDCS was safe and well tolerated, though at-home adherence and in-class attendance were variable. While they were not statistically significant, the largest effect sizes for active vs. sham tDCS were for everyday mindfulness $(d=0.6)$ and social functioning $(d=0.9)\left(F_{(1,21)}=3.68, p=0.07\right.$ and $F_{(1,21)}=3.9, p=0.06$, respectively).

Conclusions Our findings suggest that it is feasible and safe to combine tDCS with MBSR in older depressed and anxious adults, including during remote, at-home use. Furthermore, tDCS may enhance MBSR via transferring its meditative learning and practice into increases in everyday mindfulness. Future studies need to improve adherence to MBSR with tDCS.

Trial Registration ClinicalTrials.gov (NCT03653351 and NCT03680664).
\end{abstract}

Keywords Mindfulness $\cdot$ tDCS $\cdot$ MBSR $\cdot$ Cognitive function $\cdot$ Subjective cognitive complaints $\cdot$ Late-life anxiety $\cdot$ Late-life depression

Cognitive difficulties are highly prevalent in older adults with symptoms of depression or anxiety (Montejo et al.,

Tarek K. Rajji

Tarek.Rajji@camh.ca

1 Centre for Addiction and Mental Health and Department of Psychiatry, University of Toronto, Toronto, ON, Canada

2 Washington University School of Medicine, St. Louis, MO, USA

3 Centre for Mindfulness Studies, Toronto, Canada

4 Toronto Dementia Research Alliance, University of Toronto, Toronto, Canada
2011; Slavin et al., 2010). Older adults with subjective cognitive complaints are at an increased risk of progression to mild cognitive impairment (MCI) or dementia (Mitchell et al., 2014; Vilalta-Franch et al., 2013). There is also a robust association of depression and anxiety with cognitive decline (Bierman et al., 2005; Donovan et al., 2017; Johnson et al., 2015). Older adults with symptoms of depression have poorer objective global cognition (Seo et al., 2017). Conversely, subjective memory complaints can lead to depressive symptoms (Bhang et al., 2020). Taken together, these data demonstrate that older adults with cognitive complaints and mood or anxiety symptoms are at higher 
risk for cognitive decline, disability, and poor quality of life (Johnson et al., 2013; Roehr et al., 2017). As the older adult population continues to rapidly grow, a number of them may develop both memory difficulties and mood-related problems, which could cause a burden on their caregivers and the healthcare system. Older adults with memory complaints and mood symptoms comprise an important high-risk group for significant cognitive decline.

There are no evidence-based interventions for older adults that target cognitive difficulties in the context of depression or anxiety, although some recent clinical trials have shown promise for antidepressants combined with pro-cognitive interventions (Lavretsky et al., 2020; Lenze et al., 2020; Reynolds et al., 2011). Such interventions are urgently needed, as they could improve cognitive function, depressive and anxiety symptoms, and quality of life, and prevent further cognitive decline (Lenze et al., 2018). An ideal intervention would be associated with minimal burden to the individual, their family, and the healthcare system (Shalev et al., 2020).

Mindfulness is defined as a process in which one deliberately pays attention to the present moment, nonjudgmentally, and merely accepting it with an open and inquisitive nature (Kabat-Zinn, 1990). It differs from typical attention or awareness in that it involves attending to the present moment and learning to accept one's thoughts and feelings as "existing," noting that they can or cannot be true, instead of accepting these thoughts and feelings as "facts" (Baer, 2003; Faucher et al., 2016). This allows for attention to be directed towards finding solutions and methods of coping in situations perceived as stressful (Faucher et al., 2016). Mindfulness-Based Stress Reduction (MBSR (Kabat-Zinn, 1990)) is one of the most extensively researched and evaluated mindfulness-based interventions. MBSR is a group-based program focused on the systematic teaching of the conceptual elements and practice of mindfulness to enhance psychological and emotional resilience. Key concepts of mindfulness are taught and practiced in weekly groups led by a trained mindfulness instructor over a 2-month period. The other aspect of MBSR is home practice of mindfulness via meditation, ideally daily. MBSR has received considerable attention in mental health because it can reduce depressive and anxiety symptoms (Gallegos et al., 2013; Lenze et al., 2014; Moynihan et al., 2013; Wetherell et al., 2017) and improve cognitive function in older adults (Lenze et al., 2014; Moynihan et al., 2013). Additionally, MBSR may improve the regulation of attention and affect and in some areas of executive functioning (Chambers et al., 2008; Jha et al., 2007). Thus, MBSR is a promising intervention for older adults with cognitive difficulties co-occurring with depression and anxiety, provided participants adhere to the in-class attendance and at-home practice.
Transcranial direct current stimulation (tDCS) has also been shown to improve cognition in older adults (Antonenko et al., 2019; Fertonani et al., 2014; Meinzer et al., 2013, 2015). tDCS is a safe, non-invasive form of neurostimulation which delivers low levels of direct electrical current to targeted brain areas to modulate neuronal excitability and neuroplasticity (Fertonani et al., 2014). Typically, anodal stimulation depolarizes membranes and increases cortical excitability (Nitsche \& Paulus, 2000). tDCS administered over the dorsolateral prefrontal cortex (DLPFC) has been shown to improve working memory (Jones et al., 2015; Ruf et al., 2017) and word retrieval (Fertonani et al., 2014) in older adults. Furthermore, the combination of working memory training and tDCS improved working memory performance beyond working memory training alone (Teo et al., 2011). Thus, by increasing neuronal plasticity, tDCS may optimize cognitive enhancement from other behavioral interventions administered simultaneously (Hanley et al., 2020).

A substantial benefit of MBSR and tDCS is that they can be delivered remotely, which improves their reach and scalability. Fully remote capabilities of interventions have been brought to the forefront by the current COVID-19 pandemic (Nicol et al., 2020). MBSR classes are typically conducted in-person but in the wake of the COVID-19 pandemic, practitioners and research studies have moved MBSR to remote videoconference classes. Similarly, tDCS is the only neurostimulation treatment that requires no inperson contact with practitioners. MBSR involves guided practice and meditation that can be done at home. With adequate training on the use of the device and electrode placement, adults can self-administer tDCS safely and successfully (Gough et al., 2020; Kasschau et al., 2016). Both MBSR and tDCS trigger positive effects with regard to mood symptoms and cognition, and can easily be delivered remotely. However, the feasibility of combining these two interventions in older adults has not been examined. Thus, we conducted a study to assess the feasibility, safety, and tolerability of combining MBSR and tDCS in older adults with subjective cognitive complaints. We also explored whether this combination preliminarily improves cognition, mindfulness, symptoms of depression and anxiety, and social participation. As both interventions are non-invasive and low-burden to participants, the findings of this study may support the use of MBSR and tDCS to prevent cognitive decline in community-dwelling older adults with little burden to the healthcare system.

\section{Methods}

\section{Participants}

We conducted a randomized, double-blind, sham-controlled trial of tDCS combined with MBSR in adults aged 60 years or older with symptoms of depression or anxiety plus subjective cognitive complaints in two academic 
centers (Centre for Addiction and Mental Health, Toronto, ON, Canada; and Washington University in St. Louis, MO, USA). The study was approved by both institutional review boards, in which experiments were done in accordance with the Helsinki Declaration of 1975. All participants provided written informed consent.

Participants were recruited between 27 June 2018 and 6 March 2019. They completed an initial phone screen followed by an in-person screening assessment, which included a review of medical history and medications to determine eligibility. To be eligible, participants had to report cognitive complaints but have intact cognitive function as ascertained by a Short Blessed Test (Katzman et al., 1983) score $\leq 10$ and a Montreal Cognitive Assessment (MoCA; (Nasreddine et al., 2005) score $\geq 25$. Participants were included if their baseline PROMIS 8-item depression score was $\geq 16$ or their baseline PROMIS 7-item anxiety score was $\geq 14$, indicating moderate to severe symptoms.

Exclusion criteria included unstable medical illness (e.g., uncontrolled diabetes mellitus or hypertension), metal implants, significant neurological conditions (e.g. stroke, seizure disorders), use of cognitive enhancers (e.g., donepezil) within the past 6 weeks, use of anticonvulsants or antipsychotics (other than low-dose aripiprazole $(\leq 2 \mathrm{mg})$ or low-dose gabapentin $(\leq 100 \mathrm{mg})$ if prescribed for pain), concurrent cognitive training with brain training software, participation in psychotherapy, regular participation in mindfulness practice or yoga, IQ $<70$ as estimated by the Wechsler Test of Adult Reading (WTAR (Wechsler, 2001)), lifetime diagnosis of bipolar disorder, schizophrenia, schizoaffective disorder, or untreated post-traumatic stress disorder, or substance abuse within 6 months, as diagnosed by the Mini-International Neuropsychiatric Interview (MINI (Sheehan et al., 1998)). Participants meeting all eligibility criteria completed baseline assessments, which included self-report and neuropsychological assessments of outcomes, listed below.

Twenty-six participants were enrolled in the study: 14 were randomized to sham $\mathrm{tDCS}$ (mean age $=69.2, \mathrm{SD}=4.6$; nine females, five males) and 12 to active tDCS (mean age $=68.2, \mathrm{SD}=5.9$; nine females, three males). Demographic, clinical, and cognitive characteristics are presented in Table 1 . There was a difference in gender between the two groups, such that the sham group included more males.

\section{Procedure}

All participants received MBSR; they were randomly assigned (1:1) to active or sham tDCS, using a permuted block approach to ensure treatment balance within each study site. Participants, MBSR instructors, and outcome raters were blinded to the tDCS condition (i.e., active vs. sham). Following the protocol of a large trial assessing the efficacy of combing tDCS with cognitive remediation in preventing dementia among older adults with mild cognitive impairment or remitted depression (Rajji et al., 2020), active tDCS administration involved applying excitatory bilateral stimulation to the left and right DLPFC using Magstim/Neuronika HDC-Kit devices. To achieve this bilateral stimulation, the anode was placed at $\mathrm{Fz}$ and the cathode at $\mathrm{Iz}$ as per the 10-20 international electrode placement system (see Rajji et al., 2020 for electrode montage).
Table 1 Baseline demographic and clinical characteristics of participants

\begin{tabular}{llllll}
\hline Variable & Active $(n=12)$ & Sham $(n=14)$ & $t$ or $\chi^{2}$ & df & $p$-value \\
\hline Age & $68.3(5.9)$ & $69.0(5.0)$ & 0.32 & 22 & 0.75 \\
Sex (M:F) & $5: 9$ & $9: 3$ & 4.01 & 1 & 0.05 \\
Race (Caucasian:Other) & $9: 5$ & $8: 2$ & 1.43 & 3 & 0.70 \\
MoCA & $26.4(2.3)$ & $26.5(2.1)$ & 0.1 & 24 & 0.92 \\
WTAR Standard Score & $116.4(6.8)$ & $120.4(4.4)$ & 1.74 & 18.35 & 0.10 \\
NIH Toolbox Fluid Cognition Composite score & $42.5(9.32)$ & $47.5(8.2)$ & 1.42 & 22 & 0.17 \\
Word List Total Recall $^{\text {a }}$ & $26.5(5.1)$ & $27.1(5.0)$ & 0.34 & 23 & 0.74 \\
Word List Delayed Recall & $6.1(1.9)$ & $4.6(2.9)$ & -1.45 & 23 & 0.16 \\
CAMS-R & $29.8(5.7)$ & $31.1(4.7)$ & 0.65 & 24 & 0.52 \\
PROMIS Depression & $19.9(6.5)$ & $19.6(4.0)$ & -0.17 & 24 & 0.87 \\
PROMIS Anxiety & $20.1(4.6)$ & $20.7(5.2)$ & 0.33 & 24 & 0.75 \\
PROMIS Ability to Participate in Social Roles & $23.9(6.5)$ & $23.6(7.2)$ & -0.10 & 24 & 0.92 \\
PROMIS Satisfaction with Social Roles & $26.4(6.0)$ & $22.6(7.7)$ & -1.38 & 24 & 0.18 \\
\hline
\end{tabular}

Except for education and gender, values are listed as mean (SD)

$t$, independent-samples $t$-test; $\chi^{2}$, Chi-square test; $d f$, degrees of freedom; MoCA, Montreal Cognitive Assessment; WTAR, Wechsler Test of Adult Reading; PROMIS, Patient-Reported Outcomes Measurement Information System; NIH, National Institute of Health; CAMS-R, Cognitive and Affective Mindfulness Scale

${ }^{a} T$ score corrected for age, education, gender, and race; standardized based on a mean of 50 and a SD of 10 
Rubber electrodes $(5 \times 5 \mathrm{~cm})$ were inserted into salinesoaked sponges $(5 \times 7 \mathrm{~cm})$ and fixed in place using a mesh cap. The machine ramps up over the course of $30 \mathrm{~s}$ at the beginning of stimulation, and ramps down over $30 \mathrm{~s}$ at the end of stimulation in both active and sham groups. The tDCS was applied using a direct current of $2 \mathrm{~mA}$ (current density of $0.57 \mathrm{~A} / \mathrm{m}^{2}$ ) for $30 \mathrm{~min}$ per day (Rajji et al., 2020) during meditative practices of MBSR. The same parameters were used for sham stimulation, except the device shuts off after $3 \%$ of the active stimulation time, resulting in $54 \mathrm{~s}$ of active stimulation. This brief period of active stimulation is not known to produce active neurological effects, but does ensure blinding because it is associated with common side effects of active tDCS, such as tingling and itching under the electrodes (Dinn et al., 2017; Gandiga et al., 2006; Valiengo et al., 2020). For a full description of the tDCS parameters, please see Rajji et al. (2020).

Prior to the initiation of the intervention, all participants completed tDCS training provided at the local academic hospital using a group format. During five 1-h group sessions provided over five consecutive days, participants were given an overview of the tDCS device and detailed instructions for administration, programming, resetting, electrode placement, and safety procedures (such as aborting sessions safely). They were also trained on how to troubleshoot tDCS problems, such as managing side effects, e.g., tingling and itching, and self-recording necessary data. At the end of training, participants were required to demonstrate their competency self-administering tDCS by passing an assessment administered by the study coordinators. Throughout the tDCS training sessions and the intervention trial, participants had access to a training video detailing the procedures to guide them through tDCS setup and administration, as needed. A manual and coordinator contact information were also provided to each participant, and the study coordinator was available to troubleshoot over the phone during at-home tDCS sessions.

The MBSR training was based on the program developed by Jon Kabat-Zinn at the Center for Mindfulness University of Massachusetts Medical School (Lenze et al., 2014; Wetherell et al., 2017). The in-class MBSR training was led by qualified MBSR instructors, and was conducted at the academic hospital using a group format over approximately 10 weeks; group sessions included an orientation session, 8 weekly 2.5 -h classes, with a one half-day silent retreat between classes 6 and 7. This day involves a series of guided meditations and seamless mindfulness practice, and tDCS was applied for $30 \mathrm{~min}$ of the silent retreat. All participants were required to attend the MBSR in-person classes and instructed to refrain from discussing tDCS treatment among themselves to protect blinding. Content included instruction in mindfulness meditation, mindful movements including hatha yoga and walking meditation, body scans, and discussion of home practices to enhance mindfulness in everyday life. Additionally, in-class specific exercises such as identifying pleasant, unpleasant, or neutral thoughts, emotions, and body sensations are included. Poetry and teaching stories are sometimes used during class to connect participants emotionally. The qualified MBSR instructor aims to embody a mindfulness stance by the way by inquiring into participants' experiences and conducting whole or small group discussions throughout the sessions (Dobkin et al., 2014).

At the end of each in-class session, the qualified MBSR instructor would give the group a structured 30 min MBSR activity (e.g., formal practice) and associated audio files to complete at home for the week. Participants were asked to complete daily tDCS at the same time as the formal practice. In addition, participants were given small informal mindfulness practices to try incorporating into their everyday lives whenever they could, such as mindful walking or mindful eating. The study used A Mindfulness-Based Stress Reduction Workbook as a companion guide (Stahl et al., 2014). In summary, participants attended weekly in-class group MBSR plus tDCS sessions at the hospital, and then were asked to complete at least $30 \mathrm{~min}$ of guided MBSR and tDCS at home the other 6 days of the week.

\section{Measures}

Trained research staff blinded to participants' tDCS assignment performed pre- and post-intervention assessments. Post-intervention assessments were performed within 2 weeks of completing the intervention. Feasibility was assessed by examining participants' in-class attendance (i.e., number of in-person MBSR group sessions attended) and adherence to at-home mindfulness practice while using tDCS. Participants completed self-report $\operatorname{logs}$ daily, detailing their at-home use of tDCS and the frequency and duration of MBSR practice; these selfreport logs were reviewed by study staff weekly during the in-person group sessions, and were used to determine the at-home adherence rates. For an at-home session to be considered "complete," participants must have completed $80 \%$ of either tDCS or MBSR, or both on a given day (e.g., at least 24 min of formal practice). This cutoff was determined based on MBSR guidelines which indicate optimal adherence is attendance at least $8 / 10$ classes; in addition, $80 \%$ is a traditional benchmark for adequate adherence in clinical trials. To determine the at-home adherence rate, we first determined how many at-home sessions each participant completed by looking through their logs. We then calculated the total expected number of sessions that should have been completed at home over the period of the intervention. Lastly, we divided the total number of sessions each participant completed by the total 
number of expected sessions to get a percentage of athome adherence.

Participants also documented daily any failures of the tDCS device during stimulation and any adverse events (AEs). Participants were told during the in-class training phase of tDCS, things that might constitute an AE, and were instructed to record any potential AEs in their at-home $\operatorname{logs}$ and inform a member of the study team. A member of the study team went through the recorded AEs during the weekly in-class MBSR sessions and determined their relatedness, severity, and duration, clarifying with participants as needed. AEs that required the discontinuation of tDCS or MBSR were identified, as were serious adverse events (SAEs) defined as AEs that resulted in life-threatening health issues, hospitalization, or death.

The main cognitive outcome measure was the Fluid Cognition Composite from the National Institutes of Health (NIH) Toolbox Cognition Battery (Weintraub et al., 2013), a computer-based instrument measuring fluid cognition. In addition, immediate memory and delayed recall were measured using a standard 16-word list validated at the Washington University Alzheimer's Disease Research Center that is sensitive to change and does not have ceiling effects in preliminary studies (Lenze et al., 2014; Wetherell et al., 2017). After the word list is read to the participant, the participant is asked to repeat as many words as they can remember, in any order. Following the initial recall trial, the list is repeated three more times (a total of four times), and after each repetition, the participant is asked to recall as many words as they can remember. After a 20-min delay, the participant is asked to recall as many words from the list as they can remember.

The Cognitive Affective Mindfulness Scale - Revised (CAMS-R (Feldman et al., 2005)) was used to measure the four domains of mindfulness-attention, present-focus, awareness, and acceptance. CAMS-R is a self-report measure of everyday mindfulness that is not specific to any one type of meditation. CAMS-R asks questions about translating and integrating the domains of mindfulness into everyday life, for example, being able to focus on the present moment, and keeping track of one's thoughts and feelings. CAMS-R was administered pre- and post-intervention to determine how well participants integrated the teachings from MBSR into their everyday lives. The Cronbach's alpha for the CAMS-R at baseline was 0.72 , which indicates acceptable internal consistency of the scale.

The Patient Reported Outcomes Measurement Information System (PROMIS) measures were used to assess depression and anxiety over the past 7 days (Cella et al., 2010) including self-reported negative mood, views of self, social cognition, decreased positive affect, engagement, selfreported fear, anxious misery, hyperarousal, and somatic symptoms related to arousal (Pilkonis et al., 2011). In addition to the PROMIS measures of depression and anxiety, the two following PROMIS measures were used to assess satisfaction with, and ability to perform usual social roles and activities: the 8-item short form v2.9 PROMIS Scale for Satisfaction with Social Roles and Activities (Hahn et al., 2010) and the 8-item short form v2.0 PROMIS Ability to Participate in Social Roles and Activities (Hahn et al., 2010).

\section{Data Analyses}

All data were analyzed using the Statistical Program for Social Sciences (SPSS) version 25.0 (SPSS, 2018). Extreme outliers ( \pm 3 standard deviations) were removed for each time point. Data were visually inspected for normal distribution and were checked statistically with the Shapiro-Wilk test. Chi-square and independent-samples $t$-tests were used to evaluate differences between the active and sham tDCS groups on baseline demographic, clinical, and cognitive measures.

Measures of feasibility, tolerability, and safety were analyzed descriptively. While the primary goal of this pilot study was to assess feasibility, tolerability, and safety, preliminary evaluations of changes in efficacy measures were performed using repeated-measures analyses of variance (RM-ANOVAs), with randomization as the between-subjects variable, and time point (pre- or post-intervention) as the within-subjects variable. For cognition, the three dependent variables were as follows: NIH Toolbox Cognition Battery Fluid Cognition Composite, total recall score of the word list, and the delayed recall score of the word list. The preliminary effects of the intervention on subjective changes were explored with the following five dependent variables: CAMS-R, PROMIS depression scores, PROMIS anxiety scores, PROMIS satisfaction with social roles and activities, and PROMIS ability to participate in social roles and activities. For this pilot trial, a $p<0.05$ was considered significant and no corrections were made for multiple comparisons.

\section{Results}

\section{Evaluation of Feasibility: Adherence, Safety, and Tolerability}

Two participants dropped out during the intervention, one each in the sham and active tDCS group, due to the time commitment of the study. One additional participant in the active group declined a follow-up assessment (Fig. 1). The mean at-home adherence rate of tDCS use during MBSR practice was $54 \%$ for both the active and sham tDCS groups. Of the 10 MBSR group sessions, participants in the sham group attended a mean $(\mathrm{SD})=6.9(2.3)$ sessions; those in the active groups attended 7.2 (2.9) (Table 2). 
There were no SAEs in either group. Seven of the twelve participants in the active group reported an AE: six reported AEs possibly related to tDCS (i.e., redness, itching, tingling, or headaches associated with electrode placement and the first $30 \mathrm{~s}$ of active stimulation) and three reported AEs possibly related to tDCS or MBSR, including falling asleep during MBSR practice, worsening of depression, or dizziness (Table 2). Eight of the fourteen participants in the sham group reported an AE: seven were possibly related to tDCS, and three reported AEs possibly related to tDCS or MBSR, including falling asleep during MBSR practice, worsening of depression, or fogginess. In both groups, these AEs were mild and transient. The itching and tingling generally only lasted

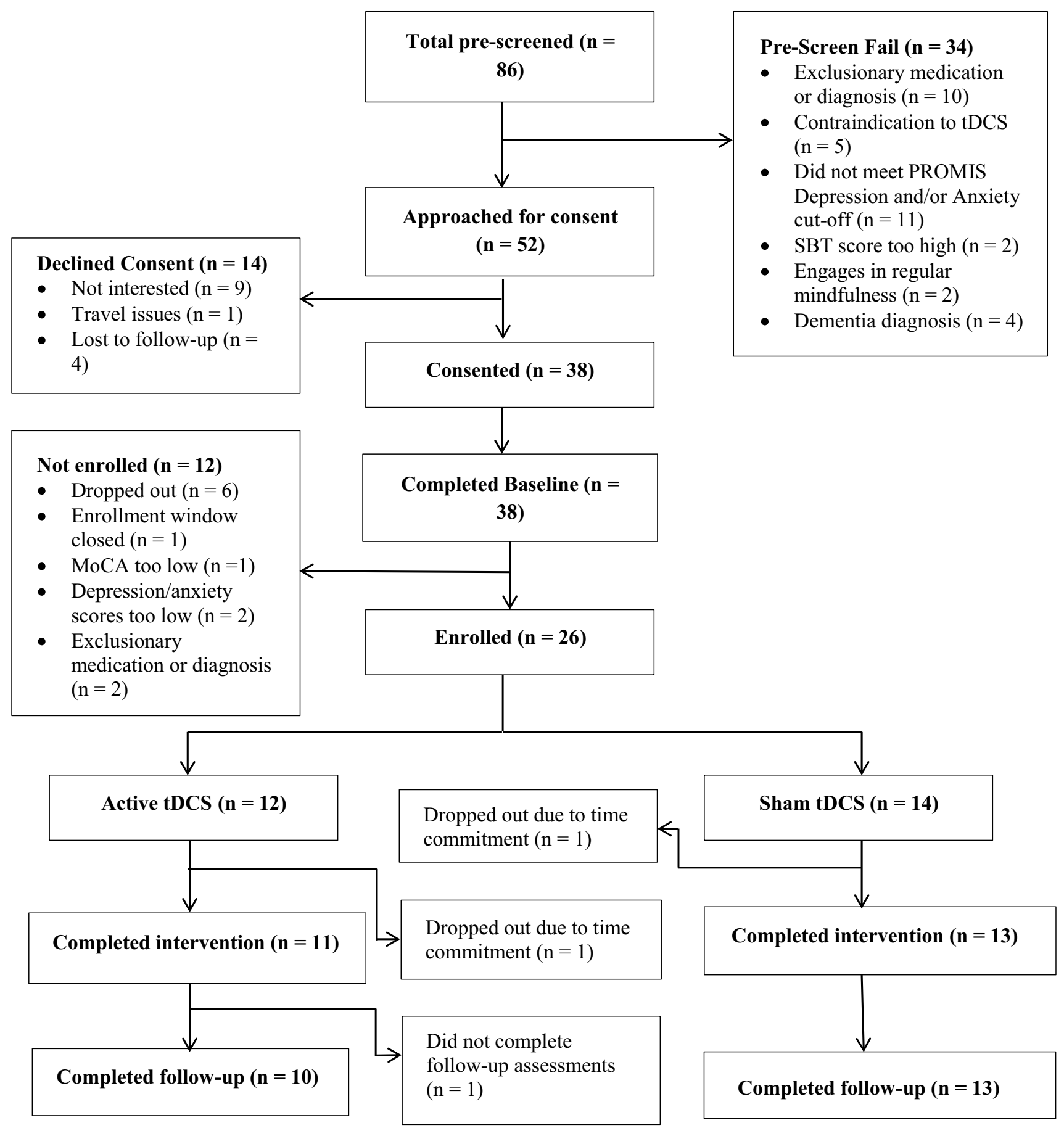

Fig. 1 Consort chart showing participant flow throughout the study 
Table 2 Feasibility data: adherence and adverse events

\begin{tabular}{|c|c|c|c|c|c|}
\hline Participant ID & Randomization group & $\begin{array}{l}\text { Rate of at-home adherence } \\
\text { with use of tDCS during } \\
\text { MBSR practice }\end{array}$ & $\begin{array}{l}\text { Number of MBSR } \\
\text { classes attended (maxi- } \\
\text { mum: 10) }\end{array}$ & $\begin{array}{l}\text { Any } A E \\
\text { reported? } \\
(\mathrm{Y} / \mathrm{N})\end{array}$ & Specific AEs reported \\
\hline & Active $t D C S$ & & & & \\
\hline 2 & & $0 \%$ & 10 & $\mathrm{~N}$ & - \\
\hline 4 & & $81 \%$ & 8 & $\mathrm{Y}$ & $\begin{array}{l}\text { Burning sensation under } \\
\text { electrode }\end{array}$ \\
\hline 6 & & $85 \%$ & 9 & $\mathrm{Y}$ & $\begin{array}{l}\text { Redness, tingling, burning sen- } \\
\text { sation on scalp, worsening of } \\
\text { depression, fatigue, headache, } \\
\text { stiffness in neck, fall }\end{array}$ \\
\hline 10 & & $88 \%$ & 7 & $\mathrm{~N}$ & - \\
\hline 11 & & $85 \%$ & 9 & $\mathrm{~N}$ & - \\
\hline 12 & & $79 \%$ & 9 & $\mathrm{Y}$ & Fell asleep \\
\hline 15 & & $62 \%$ & 10 & $\mathrm{Y}$ & $\begin{array}{l}\text { Tingling, painful sensation on } \\
\text { scalp, minor discomfort on } \\
\text { scalp }\end{array}$ \\
\hline 17 & & $0 \%^{\mathrm{a}}$ & 1 & $\mathrm{Y}$ & $\begin{array}{l}\text { Dizziness, headache, painful } \\
\text { sensation on scalp }\end{array}$ \\
\hline 19 & & $10 \%$ & 4 & $\mathrm{Y}$ & Itchiness of scalp \\
\hline 21 & & $7 \%$ & 3 & $\mathrm{~N}$ & - \\
\hline 24 & & $51 \%$ & 9 & $\mathrm{Y}$ & Redness \\
\hline 25 & & $97 \%^{\mathrm{b}}$ & 7 & $\mathrm{~N}$ & - \\
\hline \multirow[t]{2}{*}{ Mean } & & $54 \%$ & 7.2 & $7 / 12$ & \\
\hline & Sham tDCS & & & & \\
\hline 1 & & $87 \%$ & 10 & $\mathrm{Y}$ & Worsening of depression \\
\hline 3 & & $83 \%$ & 6 & $\mathrm{~N}$ & - \\
\hline 5 & & $34 \%$ & 6 & $\mathrm{~N}$ & - \\
\hline 7 & & $91 \%$ & 5 & $\mathrm{Y}$ & $\begin{array}{l}\text { Fogginess, worsening of } \\
\text { depression, tingling on scalp, } \\
\text { redness on scalp, difficulty } \\
\text { sleeping }\end{array}$ \\
\hline 8 & & $50 \%$ & 9 & $\mathrm{Y}$ & $\begin{array}{l}\text { Tingling and burning sensation } \\
\text { under electrodes }\end{array}$ \\
\hline 9 & & $35 \%^{\mathrm{c}}$ & 4 & $\mathrm{~N}$ & - \\
\hline 13 & & $29 \%$ & 4 & $\mathrm{~N}$ & - \\
\hline 14 & & $40 \%$ & 6 & $\mathrm{Y}$ & Tingling on scalp, headache \\
\hline 16 & & $59 \%$ & 9 & $\mathrm{Y}$ & Tingling on scalp, shocks \\
\hline 18 & & $38 \%$ & 9 & $\mathrm{Y}$ & $\begin{array}{l}\text { Pressure at temples, headache, } \\
\text { falling asleep }\end{array}$ \\
\hline 20 & & $28 \%$ & 3 & $\mathrm{~N}$ & - \\
\hline 22 & & $11 \%$ & 8 & $\mathrm{~N}$ & - \\
\hline 23 & & $77 \%$ & 9 & $\mathrm{Y}$ & Tingling on scalp \\
\hline 26 & & $46 \%$ & 8 & $\mathrm{Y}$ & Tingling on scalp \\
\hline Mean & & $54 \%$ & 6.9 & $8 / 14$ & \\
\hline
\end{tabular}

$\dagger$ Rate of adherence defined as the total number of sessions of combined MBSR + tDCS completed at home, divided by the total number of sessions that could have been completed while they were in the study

a Dropped out of study after MBSR class1; rate of adherence is for the time they were in the intervention

${ }^{b}$ Dropped out of study after MBSR class 7; rate of adherence is for the time they were in the intervention

${ }^{\mathrm{c}}$ Dropped out of study after MBSR class 5; rate of adherence is for the time they were in the intervention

$A E$, adverse event 
Table 3 Changes in outcome measures during the study

\begin{tabular}{|c|c|c|c|c|c|c|c|}
\hline \multirow[t]{2}{*}{ Outcome variable } & \multicolumn{2}{|l|}{ Active } & \multicolumn{2}{|l|}{ Sham } & \multirow[b]{2}{*}{ Cohen's $d$} & \multirow{2}{*}{$\begin{array}{l}\text { Time } \\
F(d f), p\end{array}$} & \multirow{2}{*}{$\begin{array}{l}\text { Time } \times \text { group } \\
F(d f), p\end{array}$} \\
\hline & Baseline & Follow-up & Baseline & Follow-up & & & \\
\hline NIH Toolbox Fluid Cognition Composite score & $42.5(9.3)$ & $47.7(8.6)$ & $47.5(8.3)$ & $49.8(10.7)$ & 0.2 & $17.1(1,20), 0.001$ & $1.8(1,20), 0.19$ \\
\hline Word List Total Recall & $26.5(5.1)$ & $26.3(7.8)$ & $27.1(5.0)$ & $28.5(6.8)$ & 0.3 & $0.03(1,20), 0.87$ & $1.1(1,20), 0.31$ \\
\hline Word List Delayed Recall & $6.67(1.5)$ & $5.0(2.8)$ & $4.46(2.9)$ & $5.54(3.2)$ & 0.2 & $0.16(1,20), 0.70$ & $3.38(1,20), 0.08$ \\
\hline CAMS-R & $29.8(5.7)$ & $34.3(4.2)$ & $31.1(4.7)$ & $32.0(4.2)$ & 0.6 & $12.2(1,21), 0.002$ & $3.7(1,21), 0.07$ \\
\hline PROMIS Depression & $19.9(6.5)$ & $15.4(7.2)$ & $19.6(4.0)$ & $16.2(4.3)$ & 0.1 & $3.8(1,21), 0.06$ & $2.2(1,21), 0.15$ \\
\hline PROMIS Anxiety & $20.1(4.6)$ & $16.3(5.9)$ & $20.7(5.2)$ & $19.2(4.0)$ & 0.6 & $7.1(1,21), 0.02$ & $0.04(1,21), 0.84$ \\
\hline PROMIS Ability to Participate in Social Roles & $23.9(6.4)$ & $27.9(5.6)$ & $23.6(7.2)$ & $22.4(6.5)$ & 0.9 & $0.4(1,21), 0.55$ & $3.9(1,21), 0.06$ \\
\hline PROMIS Satisfaction with Social Roles & $26.4(6.0)$ & $27.1(6.4)$ & $22.6(7.7)$ & $22.0(6.8)$ & 0.8 & $0.4(1,21), 0.54$ & $0.4(1,21), 0.54$ \\
\hline
\end{tabular}

$F$, repeated-measures ANOVA; $d f$, degrees of freedom; all pre- and post-intervention data presented as mean (standard deviation); $N I H$, National Institute of Health; PROMIS, Patient-Reported Outcomes Measurement Information System; CAMS-R, Cognitive and Affective Mindfulness Scale

for the first few minutes of stimulation and, if needed, more saline $(\sim 1-2 \mathrm{~mL}$, as needed) was added to the sponges to alleviate these sensations. The reported headaches typically lasted for the duration of stimulation, and in rare cases, for a few hours post-stimulation. In these cases, participants were advised to take pain medication (ibuprofen, acetaminophen, etc.). The falling asleep and fogginess tended to last throughout the MBSR practice, and participants were instructed to practice sitting up versus lying down. One participant in the active group reported worsening of depression requiring antidepressant medication adjustment during the intervention phase.

\section{Exploratory Assessment of the Efficacy of the Intervention}

Results of the exploratory efficacy analyses are presented in Table 3. At the end of the intervention, the effect size between active and sham groups for the Fluid Cognition Composite was small (Cohen's $d=0.2$ ), favoring active tDCS. However, the time $\times$ group effects did not reach statistical significance for either the Fluid Cognition Composite
$\left(F_{(1,20)}=1.8, p=0.19\right)$ or the immediate $\left(F_{(1,20)}=1.1\right.$, $p=0.3)$ and delayed recall measures $\left(F_{(1,20)}=3.4, p=0.08\right.$; see supplemental Fig. 1).

The effect size for the CAMS-R (i.e., everyday mindfulness) was medium (Cohen's $d=0.6$ ) favoring active tDCS. However, the time $\times$ group effect for the CAMS-R failed to reach statistical significance $\left(F_{(1,21)}=3.7, p=0.07\right)$. In addition, there was a main effect of time on mindfulness $\left(F_{(1,21)}=12.1, p=0.002\right)$ indicating that everyday mindfulness increased overall in the sample (see Fig. 2).

The effect size for the PROMIS anxiety scores was medium (Cohen's $d=0.6$ ), favoring active tDCS, and the effect size for the PROMIS depression scores was small (Cohen's $d=0.1$ ), favoring active tDCS. However, the time $\times$ group effects were not statistically significant for the measures of anxiety $\left(F_{(1,21)}=0.04, p=0.84\right)$ or depression $\left(F_{(1,21)}=2.2, p=0.15\right.$; see supplemental figure $)$.

Finally, the effect size for the PROMIS measure of ability to participate in social roles and activities was large (Cohen's $d=0.9$ ), favoring active tDCS (see Fig. 2), and the effect size for the PROMIS measure of satisfaction with social roles was also large (Cohen's $d=0.8$ ), favoring active tDCS
Fig. 2 Changes in mindfulness and social functioning. Line diagrams showing changes in scores on a the CAMS-R and b the PROMIS Ability to Participate in Social Roles in the active and sham tDCS groups across baseline and follow-up (x-axis). Bars represent 95\% confidence intervals
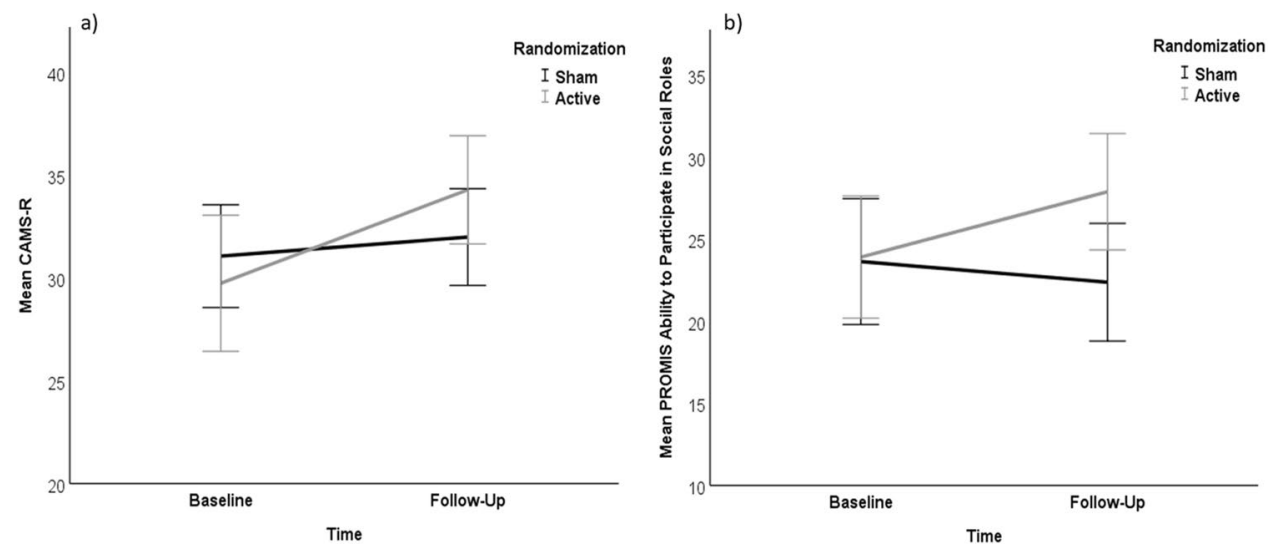
(see supplemental figure). However, the time $\times$ group effect failed to reach statistical significance for either PROMIS social measures (ability: $F_{(1,21)}=3.9, p=0.06$; satisfaction: $\left.F_{(1,21)}=0.4, p=0.5\right)$.

\section{Discussion}

We examined the feasibility, tolerability/safety, and preliminary efficacy of combining tDCS with MBSR in older adults with depression or anxiety, and subjective cognitive complaints. The three main findings indicate that (1) older adults could self-administer tDCS and practice MBSR at home; (2) this combination was safe and well-tolerated; (3) and adherence was quite variable among participants and possibly too low for achieving full benefits from the intervention. Exploratory analyses showed that the combined intervention was associated with medium to large effect sizes for reduction in anxiety, increase in everyday mindfulness, and improvement in social functioning, with smaller effect sizes for reduction in depression and improvement in cognitive performance. However, none of these differences reached statistical significance.

Overall, this feasibility study has promising findings that justify further research into tDCS-facilitated MBSR. However, it also identifies challenges that need to be addressed before such a trial should be conducted. The adherence with both at-home practice and in-class attendance at the hospital was largely variable among participants. In four previous studies using home-based tDCS, adherence rates have been reported to be at or above 80\% (Gough et al., 2020; Kasschau et al., 2016; Martens et al., 2018; Riggs et al., 2018). However, these studies had a shorter duration of at-home tDCS self-administration (e.g., 10 days), included younger individuals, or involved remote supervision (Gough et al., 2020). We believe that adherence could be improved in older patients by improving the user interface and including more individualized tDCS training. Many participants shared that they found the tDCS setup burdensome and complex, which ultimately hindered their at-home practice and adherence. Using a headband with pre-placed electrodes may decrease setup time, allow for more accurate electrode placement, and decrease participant burden. Sending daily reminders via text message or email to prompt tDCS administration may also increase adherence. A smartphone app or objective reporting provided by the tDCS machine itself detailing tDCS use, rather than a self-report log, could also be used to improve the recording of tDCS parameters, such as number of minutes of stimulation, number of failures, and impedance levels.

Most of the adverse events reported in this study were mild and transient. They included tingling, itching, pain and redness on the scalp, and headaches, almost exclusively during the course of stimulation. Three participants reported worsening of their depressive symptoms; however, this did not lead them to discontinue the study. There were no safety issues associated with tDCS. Adherence with tDCS administration and in-class attendance were comparable in the sham and active groups, suggesting that active tDCS did not cause adverse effects or discomfort associated with non-adherence.

At the completion of the study, compared to baseline, participants in both the sham and active tDCS groups reported an increase in everyday mindfulness. Those in the active tDCS group showed numerically (but not statistically) higher benefits from mindfulness, i.e., higher mindfulness scores, lower anxiety scores, and higher social participation scores. This is consistent with a previous pilot study in which active tDCS enhanced meditation, calmness, and mindfulness during mindfulness meditation sessions compared to sham tDCS (Badran et al., 2017). This suggests that active tDCS has a positive and synergistic effect on learning mindfulness, such that those randomized to active tDCS were better able to learn and apply the principles taught in the MBSR sessions to their everyday lives. An increase in mindfulness could in turn lead to a reduction in depression and/or anxiety symptoms. Mindfulness meditation emphasizes paying attention to the present moment, rather than ruminating on the past or worrying about the future. Past research indicates that MBSR reduces both worrying and ruminating (Deyo et al., 2009; Jain et al., 2007; Shapiro et al., 2008). Focusing on the present moment may help individuals reduce their anxiety and worrying behavior. An increase in mindfulness could also lead to an increase in coping mechanisms, allowing individuals with anxiety or depression to feel more equipped to deal with everyday stressors (Irving et al., 2009). Studies have shown that higher levels of mindfulness after MBSR were related to lower levels of mood symptoms and stress, and that increased mindfulness predicted stress reduction (Carlson \& Brown, 2005; Matousek \& Dobkin, 2010).

Similarly, our finding of an improvement in social functioning in both groups with a numerically (but not statistically) higher improvement with MBSR combined with tDCS is consistent with previous studies in which both tDCS and MBSR separately improved social functioning (de Vibe et al., 2017; Sellaro et al., 2016). Social functioning is known to be impaired in individuals with psychiatric conditions, and when psychiatric symptoms (e.g., depression and anxiety) improve, social functioning and quality of life tend to improve as well (Furukawa et al., 2001; Lin et al., 2013; Renner et al., 2014). These findings must be interpreted cautiously, given the small sample size, lack of statistical significance, and feasibility (not efficacy) focus of the trial.

Changes in our three cognitive measures (NIH Toolbox Cognition Battery Fluid Cognition Composite, total or delayed word recall) did not differ significantly between the two groups. Ceiling effects were observed on some of the 
measures on the NIH Toolbox Cognition Battery, such as the Flanker Inhibitory Control Test and the Dimensional Change Card Sort Test. The skills taught in MBSR are complex cognitive processes, such as directed attention and executive control. It is possible that the teaching and practice of MBSR tasks benefitted from active tDCS, allowing transfer of skills learned and practiced to everyday mindfulness as indexed by higher CAMS-R scores. This everyday mindfulness, which involves higher-order cognition, such as complex attention and executive function, is more cognitively demanding than the NIH Toolbox cognitive tasks, which may have allowed the detection of the added value of tDCS stimulation. To be better able to detect cognitive changes over time, future studies should include cognitive tests with a wider range of difficulty. These studies should also include cognitive tests that assess more complex cognitive processes to more accurately align with the complex cognitive processes involved in learning mindfulness, such as executive functioning and cognitive control. Again, these findings must be interpreted cautiously in this small feasibility study.

\section{Limitations and Future Research}

This pilot study is not without limitations. As already mentioned, the in-class attendance and at-home compliance of MBSR and tDCS had large variability among participants. However, we believe there are ways to improve and enhance both in-class attendance and at-home compliance, which were described above. It is possible that the adherence was too low in the study to achieve the full benefits of the intervention, and that with increased compliance we could see additional benefits. Although combined MBSR and tDCS is a relatively low-burden intervention compared to other interventions which can require daily trips to the hospital, for some, weekly in-person sessions and daily at-home practice might be considered burdensome and/or time-intensive. We recognize that the time commitment for the combined interventions might be a limitation for some. The self-report aspect of tDCS and MBSR compliance is also a limitation; it is possible that participants over- or under-reported their at-home tDCS and MBSR practice. Future studies should focus on having more objective measures of compliance, in addition to self-report measures, such as the session logs from the tDCS devices.

The sample size for this pilot study is also a major limitation, and thus the preliminary efficacy results should be interpreted with caution. The preliminary results, however, do indicate there could be added benefit of active tDCS as compared to sham tDCS in enhancing the effects of the MBSR intervention. Future research should continue to explore the efficacy of combined active tDCS and MBSR with larger samples, to determine if there are in fact synergistic effects of combining active tDCS and MBSR. We also did not explicitly exclude individuals with a history of ADHD, and/or physical problems that might impact cognition (e.g., vitamin deficiencies). Future research should consider excluding individuals with these conditions, as it is likely that combined MBSR and tDCS would not impact cognition in these cases. We also did not formally assess participants' expectations of mindfulness-based therapy prior to study enrollment. It is possible that expectation or beliefs about mindfulness could influence the efficacy of the intervention. Although a recent study demonstrated that neither patient expectancy nor perceived credibility of MBSR affected the efficacy of the intervention on improving cognition (Haddad et al., 2020), this might be another factor to consider in future research. Lastly, as indicated above, our choice of cognitive outcome measures may have obscured the potential effect of combined active tDCS and MBSR on cognition due to the ceiling performance on some of the tasks. Future research should aim to use more complex cognitive outcome measures, which may be more affected by the combination of MBSR and tDCS.

Supplementary Information The online version contains supplementary material available at https://doi.org/10.1007/s12671-021-01764-9.

Acknowledgements Magstim/Newronika loaned equipment for the project.

Author Contribution HB and HAO are co-first authors, listed alphabetically, and contributed equally to this work. EJL and TKR are co-senior authors of this work, listed alphabetically. EJL and TKR designed the trial. GM and JK were the qualified MBSR instructors, and adapted patient handouts and materials for the MBSR curriculum for the study. SK, DMB, LK, SS, HAO, HB, EJL and TKR contributed to the recruitment of participants. HB conducted data analyses. HB, HAO, EJL, TKR were responsible for the decision to submit the report and drafted it. All authors read, critically revised, and approved the report.

Funding This study was supported primarily by the National Institute of Mental Health (R25 MH112473 to Washington University) and the Canadian Institutes of Health Research (Canada Research Chair Grant \#: 950-230879 to the University of Toronto). Additional funding was provided by the Paula J. Clayton MD Hawthorne Foundation Research Award, the Taylor Family Institute for Innovative Psychiatric Research, and the Center for Brain Research in Mood Disorders.

Data Availability Consent was not obtained from participants to disseminate and share study data, and thus we are unable to provide the study dataset.

\section{Declarations}

Conflict of interest DMB has received research support from CIHR, NIH, Brain Canada, and the Temerty Family through the CAMH Foundation and the Campbell Family Research Institute. He received research support and in-kind equipment support for an investigator-initiated study from Brainsway Ltd. He is the site principal investigator for three sponsor-initiated studies for Brainsway Ltd. He also receives in-kind equipment support from Magventure for two investigator-initiated research. He received medication supplies for an investigator- 
initiated trial from Indivior. SK receives research support from Brain and Behavior Foundation, National institute on Ageing, BrightFocus Foundation, Brain Canada, Canadian Institute of Health Research, Centre for Ageing and Brain Health Innovation, Centre for Addiction and Mental Health, University of Toronto. He also receives equipment support from Soterix Medical. SK has received grant support from Brain Canada, NIH, Brain and Behavior Foundation (NARSAD), BrightFocus Foundation, Weston Brain Institute, Canadian Centre for Ageing and Brain Health Innovation, CAMH foundation and University of Toronto, and in-kind equipment support from Soterix Medical Inc. BHM receives research support from Brain Canada, CIHR, the CAMH Foundation, the Patient-Centered Outcomes Research Institute (PCORI), and the US National Institutes of Health (NIH); during the past three years, he has received non-financial support from Eli Lilly (medications and matching placebo for a NIH-funded clinical trial) and Pfizer (medications for a NIH-funded clinical trial), Capital Solution Design LLC (software used in a study funded by the CAMH Foundation), and HAPPYneuron (software used in a study founded by Brain Canada). EJL received in-kind equipment support for this investigator-initiated study from Magstim. He receives or has received support from National Institutes of Health (NIH), Takeda, Lundbeck, Acadia, the Taylor Family Institute for Innovative Psychiatric Research, the McKnight Brain Research Foundation, the Center for Brain Research in Mood Disorders, and PCORI. He has received consulting fees from Janssen and Jazz Pharmaceuticals. TKR has received research support from Brain Canada, Brain and Behavior Research Foundation, BrightFocus Foundation, Canada Foundation for Innovation, Canada Research Chair, Canadian Institutes of Health Research, Centre for Aging and Brain Health Innovation, National Institutes of Health, Ontario Ministry of Health and Long-Term Care, Ontario Ministry of Research and Innovation, and the Weston Brain Institute. TKR also received in-kind equipment support for this investigator-initiated study from Magstim, and in-kind research accounts from Scientific Brain Training Pro for other studies. HB, HAO, LK, SS, JK, and GM have no conflicts to disclose.

Ethical Approval The study was approved by both institutional review boards (Centre for Addiction and Mental Health, Toronto, Canada and Washington University, in St. Louis, Missouri), in which experiments were done in accord with the Helsinki Declaration of 1964, and its later amendments.

Informed Consent All participants provided written informed consent prior to their participation in the study.

\section{References}

Antonenko, D., Hayek, D., Netzband, J., Grittner, U., \& Floel, A. (2019). tDCS-induced episodic memory enhancement and its association with functional network coupling in older adults. Scientific Reports, 9(1), 2273. https://doi.org/10.1038/ s41598-019-38630-7

Badran, B. W., Austelle, C. W., Smith, N. R., Glusman, C. E., Froeliger, B., Garland, E. L., Borckardt, J. J., George, M. S., \& Short, B. (2017). A double-blind study exploring the use of transcranial direct current stimulation (tDCS) to potentially enhance mindfulness meditation (E-Meditation). Brain Stimulation: Basic, Translational, and Clinical Research in Neuromodulation, 10(1), $152-154$.

Baer, R. A. (2003). Mindfulness training as a clinical intervention: A conceptual and empirical review. Clinical Psychology: Science and Practice, 10(2), 125
Bhang, I., Mogle, J., Hill, N., Whitaker, E. B., \& Bhargava, S. (2020). Examining the temporal associations between self-reported memory problems and depressive symptoms in older adults. Aging \& Mental Health, 24(11), 1864-1871.

Bierman, E., Comijs, H., Jonker, C., \& Beekman, A. (2005). Effects of anxiety versus depression on cognition in later life. The American Journal of Geriatric Psychiatry, 13(8), 686-693.

Carlson, L. E., \& Brown, K. W. (2005). Validation of the Mindful Attention Awareness Scale in a cancer population. Journal of Psychosomatic Research, 58(1), 29-33.

Cella, D., Riley, W., Stone, A., Rothrock, N., Reeve, B., Yount, S., Amtmann, D., Bode, R., Buysse, D., \& Choi, S. (2010). The PatientReported Outcomes Measurement Information System (PROMIS) developed and tested its first wave of adult self-reported health outcome item banks: 2005-2008. Journal of Clinical Epidemiology, 63(11), 1179-1194.

Chambers, R., Lo, B. C. Y., \& Allen, N. B. (2008). The impact of intensive mindfulness training on attentional control, cognitive style, and affect. Cognitive Therapy and Research, 32(3), 303-322.

de Vibe, M., Bjørndal, A., Fattah, S., Dyrdal, G. M., Halland, E., \& Tanner-Smith, E. E. (2017). Mindfulness-based stress reduction (MBSR) for improving health, quality of life and social functioning in adults: A systematic review and meta-analysis. Campbell Systematic Reviews, 13(1), 1-264.

Deyo, M., Wilson, K. A., Ong, J., \& Koopman, C. (2009). Mindfulness and rumination: Does mindfulness training lead to reductions in the ruminative thinking associated with depression? Explore, 5(5), 265-271.

Dinn, W., Göral, F., Adigüzel, S., Karamürsel, S., Fregni, F., \& Aycicegi-Dinn, A. (2017). Effectiveness of tDCS blinding protocol in a sham-controlled study. Brain Stimulation: Basic, Translational, and Clinical Research in Neuromodulation, 10(2), 401.

Dobkin, P. L., Hickman, S., \& Monshat, K. (2014). Holding the heart of mindfulness-based stress reduction: Balancing fidelity and imagination when adapting MBSR. Mindfulness, 5(6), 710-718.

Donovan, N. J., Wu, Q., Rentz, D. M., Sperling, R. A., Marshall, G. A., \& Glymour, M. M. (2017). Loneliness, depression and cognitive function in older US adults. International Journal of Geriatric Psychiatry, 32(5), 564-573.

Faucher, J., Koszycki, D., Bradwejn, J., Merali, Z., \& Bielajew, C. (2016). Effects of CBT versus MBSR treatment on social stress reactions in social anxiety disorder. Mindfulness, 7(2), 514-526.

Feldman, G., Hayes, A., Kumar, S., Kamholz, B., Greeson, J., \& Laurenceau, J. (2005). Assessing mindfulness in the context of emotion regulation: The Revised Cognitive and Affective Mindfulness Scale (CAMS-R). In 39th Annual Convention of the Association for behavioral and cognitive therapies, Washington, DC.

Fertonani, A., Brambilla, M., Cotelli, M., \& Miniussi, C. (2014). The timing of cognitive plasticity in physiological aging: A tDCS study of naming. Frontiers in Aging Neuroscience, 6, 131.

Furukawa, T. A., Takeuchi, H., Hiroe, T., Mashiko, H., Kamei, K., Kitamura, T., \& Takahashi, K. (2001). Symptomatic recovery and social functioning in major depression. Acta Psychiatrica Scandinavica, 103(4), 257-261.

Gallegos, A. M., Hoerger, M., Talbot, N. L., Moynihan, J. A., \& Duberstein, P. R. (2013). Emotional benefits of mindfulnessbased stress reduction in older adults: The moderating roles of age and depressive symptom severity. Aging \& Mental Health, 17(7), 823-829.

Gandiga, P. C., Hummel, F. C., \& Cohen, L. G. (2006). Transcranial DC stimulation (tDCS): A tool for double-blind sham-controlled clinical studies in brain stimulation. Clinical Neurophysiology, 117(4), 845-850.

Gough, N., Brkan, L., Subramaniam, P., Chiuccariello, L., De Petrillo, A., Mulsant, B. H., Bowie, C. R., \& Rajji, T. K. (2020). Feasibility of remotely supervised transcranial direct current stimulation and 
cognitive remediation: A systematic review. PLOS ONE, 15(2), $\mathrm{e} 0223029$.

Haddad, R., Lenze, E. J., Nicol, G., Miller, J. P., Yingling, M., \& Wetherell, J. L. (2020). Does patient expectancy account for the cognitive and clinical benefits of mindfulness training in older adults? International Journal of Geriatric Psychiatry, 35(6), 626-632.

Hahn, E. A., DeVellis, R. F., Bode, R. K., Garcia, S. F., Castel, L. D., Eisen, S. V., Bosworth, H. B., Heinemann, A. W., Rothrock, N., \& Cella, D. (2010). Measuring social health in the patient-reported outcomes measurement information system (PROMIS): Item bank development and testing. Quality of Life Research, 19(7), $1035-1044$.

Hanley, C. J., Alderman, S. L., \& Clemence, E. (2020). Optimising cognitive enhancement: Systematic assessment of the effects of tdcs duration in older adults. Brain Sciences, 10(5), 304.

Irving, J. A., Dobkin, P. L., \& Park, J. (2009). Cultivating mindfulness in health care professionals: A review of empirical studies of mindfulness-based stress reduction (MBSR). Complementary Therapies in Clinical Practice, 15(2), 61-66.

Jain, S., Shapiro, S. L., Swanick, S., Roesch, S. C., Mills, P. J., Bell, I., \& Schwartz, G. E. (2007). A randomized controlled trial of mindfulness meditation versus relaxation training: Effects on distress, positive states of mind, rumination, and distraction. Annals of Behavioral Medicine, 33(1), 11-21.

Jha, A. P., Krompinger, J., \& Baime, M. J. (2007). Mindfulness training modifies subsystems of attention. Cognitive, Affective, \& Behavioral Neuroscience, 7(2), 109-119.

Johnson, L. A., Mauer, C., Jahn, D., Song, M., Wyshywaniuk, L., Hall, J. R., Balldin, V. H., \& O'Bryant, S. E. (2013). Cognitive differences among depressed and non-depressed MCI participants: A project FRONTIER study. International Journal of Geriatric Psychiatry, 28(4), 377-382.

Johnson, L. A., Sohrabi, H. R., Hall, J. R., Kevin, T., Edwards, M., O'Bryant, S. E., \& Martins, R. N. (2015). A depressive endophenotype of poorer cognition among cognitively healthy community-dwelling adults: Results from the Western Australia memory study. International Journal of Geriatric Psychiatry, 30(8), 881-886.

Jones, K. T., Stephens, J. A., Alam, M., Bikson, M., \& Berryhill, M. E. (2015). Longitudinal neurostimulation in older adults improves working memory. PLoS ONE, 10(4), e0121904.

Kabat-Zinn, J. (1990). Full catastrophe living: Using the wisdom of your body and mind to face stress, pain and illness. Delta.

Kasschau, M., Reisner, J., Sherman, K., Bikson, M., Datta, A., \& Charvet, L. E. (2016). Transcranial direct current stimulation is feasible for remotely supervised home delivery in multiple sclerosis. Neuromodulation: Technology at the Neural Interface, 19(8), 824-831.

Katzman, R., Brown, T., Fuld, P., Peck, A., Schechter, R., \& Schimmel, H. (1983). Validation of a short Orientation-Memory-Concentration Test of cognitive impairment. The American Journal of Psychiatry, 140(6), 734-739.

Lavretsky, H., Laird, K. T., Krause-Sorio, B., Heimberg, B. F., Yeargin, J., Grzenda, A., Wu, P., Thana-Udom, K., Ercoli, L. M., \& Siddarth, P. (2020). A randomized double-blind placebo-controlled trial of combined escitalopram and memantine for older adults with major depression and subjective memory complaints. The American Journal of Geriatric Psychiatry, 28(2), 178-190.

Lenze, E. J., Hickman, S., Hershey, T., Wendleton, L., Ly, K., Dixon, D., Doré, P., \& Wetherell, J. L. (2014). Mindfulness-based stress reduction for older adults with worry symptoms and co-occurring cognitive dysfunction. International Journal of Geriatric Psychiatry, 29(10), 991-1000.

Lenze, E. J., Stevens, A., Waring, J. D., Pham, V. T., Haddad, R., Shimony, J., Miller, J. P., \& Bowie, C. R. (2020). Augmenting computerized cognitive training with vortioxetine for age-related cognitive decline: A randomized controlled trial. American Journal of Psychiatry, 177(6), 548-555.

Lenze, E. J., Voineskos, A. N., Butters, M. A., \& Karp, J. F. (2018). Stopping cognitive decline in patients with late-life depression: A new front in the fight against dementia. The American Journal of Geriatric Psychiatry, 26(8), 828-834.

Lin, C.-H., Yen, Y.-C., Chen, M.-C., \& Chen, C.-C. (2013). Relief of depression and pain improves daily functioning and quality of life in patients with major depressive disorder. Progress in NeuroPsychopharmacology and Biological Psychiatry, 47, 93-98.

Martens, G., Lejeune, N., O’Brien, A. T., Fregni, F., Martial, C., Wannez, S., Laureys, S., \& Thibaut, A. (2018). Randomized controlled trial of home-based 4-week tDCS in chronic minimally conscious state. Brain Stimulation, 11(5), 982-990.

Matousek, R., \& Dobkin, P. L. (2010). Weathering storms: A cohort study of how participation in a mindfulness-based stress reduction program benefits women after breast cancer treatment. Current Oncology, 17(4), 62-70.

Meinzer, M., Lindenberg, R., Antonenko, D., Flaisch, T., \& Flöel, A. (2013). Anodal transcranial direct current stimulation temporarily reverses age-associated cognitive decline and functional brain activity changes. Journal of Neuroscience, 33(30), 12470-12478.

Meinzer, M., Lindenberg, R., Phan, M. T., Ulm, L., Volk, C., \& Flöel, A. (2015). Transcranial direct current stimulation in mild cognitive impairment: Behavioral effects and neural mechanisms. Alzheimer's \& Dementia, 11(9), 1032-1040.

Mitchell, A., Beaumont, H., Ferguson, D., Yadegarfar, M., \& Stubbs, B. (2014). Risk of dementia and mild cognitive impairment in older people with subjective memory complaints: A meta-analysis. Acta Psychiatrica Scandinavica, 130(6), 439-451.

Montejo, P., Montenegro, M., Fernández, M. A., \& Maestu, F. (2011). Subjective memory complaints in the elderly: Prevalence and influence of temporal orientation, depression and quality of life in a population-based study in the city of Madrid. Aging \& Mental Health, 15(1), 85-96.

Moynihan, J. A., Chapman, B. P., Klorman, R., Krasner, M. S., Duberstein, P. R., Brown, K. W., \& Talbot, N. L. (2013). Mindfulnessbased stress reduction for older adults: Effects on executive function, frontal alpha asymmetry and immune function. $\mathrm{Neu}$ ropsychobiology, 68(1), 34-43.

Nasreddine, Z. S., Phillips, N. A., Bédirian, V., Charbonneau, S., Whitehead, V., Collin, I., Cummings, J. L., \& Chertkow, H. (2005). The Montreal Cognitive Assessment, MoCA: A brief screening tool for mild cognitive impairment. Journal of the American Geriatrics Society, 53(4), 695-699.

Nicol, G. E., Piccirillo, J. F., Mulsant, B. H., \& Lenze, E. J. (2020). Action at a distance: Geriatric research during a pandemic. Journal of the American Geriatrics Society, 68(5), 922-925.

Nitsche, M. A., \& Paulus, W. (2000). Excitability changes induced in the human motor cortex by weak transcranial direct current stimulation. The Journal of Physiology, 527(3), 633-639.

Pilkonis, P. A., Choi, S. W., Reise, S. P., Stover, A. M., Riley, W. T., Cella, D., \& Group, P. C. (2011). Item banks for measuring emotional distress from the Patient-Reported Outcomes Measurement Information System (PROMIS $\left.{ }^{\circledR}\right)$ : Depression, anxiety, and anger. Assessment, 18(3), 263-283.

Rajji, T. K., Bowie, C. R., Herrmann, N., Pollock, B. G., Bikson, M., Blumberger, D. M., Butters, M. A., Daskalakis, Z. J., Fischer, C. E., \& Flint, A. J. (2020). Design and rationale of the PACt-MD randomized clinical trial: Prevention of Alzheimer's dementia with cognitive remediation plus transcranial direct current stimulation in mild cognitive impairment and depression. Journal of Alzheimer's Disease, 76(2), 733-751.

Renner, F., Cuijpers, P., \& Huibers, M. (2014). The effect of psychotherapy for depression on improvements in social functioning: A meta-analysis. Psychological Medicine, 44(14), 2913-2926. 
Reynolds, C. F., Butters, M. A., Lopez, O., Pollock, B. G., Dew, M. A., Mulsant, B. H., Lenze, E. J., Holm, M., Rogers, J. C., \& Mazumdar, S. (2011). Maintenance treatment of depression in old age: A randomized, double-blind, placebo-controlled evaluation of the efficacy and safety of donepezil combined with antidepressant pharmacotherapy. Archives of General Psychiatry, 68(1), 51-60.

Riggs, A., Patel, V., Paneri, B., Portenoy, R. K., Bikson, M., \& Knotkova, H. (2018). At-home transcranial direct current stimulation (tDCS) with telehealth support for symptom control in chronically-ill patients with multiple symptoms. Frontiers in Behavioral Neuroscience, 12, 93.

Roehr, S., Luck, T., Pabst, A., Bickel, H., König, H.-H., Lühmann, D., Fuchs, A., Wolfsgruber, S., Wiese, B., \& Weyerer, S. (2017). Subjective cognitive decline is longitudinally associated with lower health-related quality of life. International Psychogeriatrics, 29(12), 1939-1950.

Ruf, S. P., Fallgatter, A. J., \& Plewnia, C. (2017). Augmentation of working memory training by transcranial direct current stimulation (tDCS). Scientific Reports, 7(1), 1-11.

Sellaro, R., Nitsche, M. A., \& Colzato, L. S. (2016). The stimulated social brain: Effects of transcranial direct current stimulation on social cognition. Annals of the New York Academy of Sciences, 1369(1), 218-239.

Seo, E. H., Kim, H., Choi, K. Y., Lee, K. H., \& Choo, I. H. (2017). Association of subjective memory complaint and depressive symptoms with objective cognitive functions in prodromal Alzheimer's disease including pre-mild cognitive impairment. Journal of Affective Disorders, 217, 24-28.

Shalev, D., Docherty, M., Spaeth-Rublee, B., Khauli, N., Cheung, S., Levenson, J., \& Pincus, H. A. (2020). Bridging the behavioral health gap in serious illness care: Challenges and strategies for workforce development. The American Journal of Geriatric Psychiatry, 28(4), 448-462.

Shapiro, S. L., Oman, D., Thoresen, C. E., Plante, T. G., \& Flinders, T. (2008). Cultivating mindfulness: Effects on well-being. Journal of Clinical Psychology, 64(7), 840-862.

Sheehan, D. V., Lecrubier, Y., Sheehan, K. H., Amorim, P., Janavs, J., Weiller, E., Hergueta, T., Baker, R., \& Dunbar, G. C. (1998). The Mini-International Neuropsychiatric Interview (MINI): The development and validation of a structured diagnostic psychiatric interview for DSM-IV and ICD-10. Journal of Clinical Psychiatry, 59(20), 22-33.
Slavin, M. J., Brodaty, H., Kochan, N. A., Crawford, J. D., Trollor, J. N., Draper, B., \& Sachdev, P. S. (2010). Prevalence and predictors of "subjective cognitive complaints" in the Sydney Memory and Ageing Study. The American Journal of Geriatric Psychiatry, 18(8), 701-710.

SPSS, I. (2018). IBM statistical package for social services (Version 25). IBM.

Stahl, B., Meleo-Meyer, F., \& Koerbel, L. (2014). A mindfulnessbased stress reduction workbook for anxiety. New Harbinger Publications.

Teo, F., Hoy, K. E., Daskalakis, Z. J., \& Fitzgerald, P. B. (2011). Investigating the role of current strength in tDCS modulation of working memory performance in healthy controls. Frontiers in Psychiatry, 2, 45.

Valiengo, L. C. L., Goerigk, S., Gordon, P. C., Padberg, F., Serpa, M. H., Koebe, S., Dos Santos, L. A., Lovera, R. A. M., de Carvalho, J. B., \& van de Bilt, M. (2020). Efficacy and safety of transcranial direct current stimulation for treating negative symptoms in schizophrenia: A randomized clinical trial. JAMA Psychiatry, $77(2), 121-129$.

Vilalta-Franch, J., López-Pousa, S., Llinàs-Reglà, J., Calvó-Perxas, L., Merino-Aguado, J., \& Garre-Olmo, J. (2013). Depression subtypes and 5-year risk of dementia and Alzheimer disease in patients aged 70 years. International Journal of Geriatric Psychiatry, 28(4), 341-350.

Wechsler, D. (2001). Wechsler test of adult reading: WTAR. Psychological Corporation.

Weintraub, S., Dikmen, S. S., Heaton, R. K., Tulsky, D. S., Zelazo, P. D., Bauer, P. J., Carlozzi, N. E., Slotkin, J., Blitz, D., \& WallnerAllen, K. (2013). Cognition assessment using the NIH Toolbox. Neurology, 80(11 Supplement 3), S54-S64.

Wetherell, J. L., Hershey, T., Hickman, S., Tate, S. R., Dixon, D., Bower, E. S., \& Lenze, E. J. (2017). Mindfulness-based stress reduction for older adults with stress disorders and neurocognitive difficulties: A randomized controlled trial. The Journal of Clinical Psychiatry, 78(7), e734-e743.

Publisher's Note Springer Nature remains neutral with regard to jurisdictional claims in published maps and institutional affiliations. 